\section{Versatility of the cone beam computed tomography in oral surgery: an overview}

\section{Kishan G. Panicker, ${ }^{1}$ \\ Anuroopa Pudukulangara Nair, ${ }^{2}$ \\ Bipin Chandra Reddy ${ }^{1}$}

'Department of Oral and Maxillofacial

Surgery, The Oxford Dental College;

2Department of Periodontics,

Rajarajeshwari dental college \& hospital, Bangalore, India

\section{Abstract}

Cone beam CT (CBCT) produces threedimensional information on the facial skeleton, teeth and their surrounding tissues; and is increasingly being used in many of the dental specialties. This is usually achieved with a substantially lower effective dose compared with conventional medical computed tomography (CT). Periapical pathologies, root fractures, root canal anatomy and the true nature of the alveolar bone topography around teeth may be assessed. CBCT scans are desirable to assess posterior teeth prior to periapical surgery, as the thickness of the cortical and cancellous bone can be accurately determined as can the inclination of roots in relation to the surrounding jaw. The relationship of anatomical structures such as the maxillary sinus and inferior dental nerve to the root apices may also be clearly visualized. Measurements on CBCT are more accurate when compared with OPG. Therefore, CBCT permits the clinician to have all necessary information when planning dental implants. The purpose of this article is to provide an overview of the unique image display capabilities of maxillofacial CBCT systems and to illustrate specific applications in clinical practice.

\section{Introduction}

Dental imaging is an important diagnostic adjunct to the clinical assessment of dental patient. After introduction of panoromic radiography in 1960s, dental radiology had become a field of immense progress and enabled clinicians to see jaw and maxillofacial structures with a single radiography. How $\neg$ ever, two dimensional (2D) images with intraoral and extraoral procedures of three dimensional (3D) maxil-lofacial regions have some drawbacks such as magnifi $\neg$ cation and superimposition. To eliminate this drawbacks 3D imaging techniques have been developed.
Subsequent period to digital imaging, computed tomography (CT), magnetic resonance imaging (MRI), positron emission tomography (PET) and cone beam computed tomogra $\neg$ phy (CBCT) had been developed. ${ }^{1}$

Cone beam CT (CBCT) was first developed for use in angiography. In 1998, Mozzo et al. ${ }^{2}$ reported the first CBCT unit developed specifically for dental use, the NewTom 9000 (Quantitative Radiology, Verona, Italy). Other similar devices introduced at around that time included the Ortho-CT, which was renamed the 3DX (J. Morita Mfg Corp, Kyoto, Japan) multiimage micro-CT in 2000. ${ }^{3,4}$ In 2003, Hashimoto et al. ${ }^{4}$ reported that the 3DX CBCT produced better image quality with a much lower radiation dose than the newest multidetector row helical CT unit (1.19 mSv vs $458 \mathrm{mSv}$ per examination).

CBCT is capable of providing clear, submilimeter resolution images at shorter scan times, lower patient dose and lower cost compared with medical CT. Increasing availability of this technology provides the clinician's 3D representation of maxillofacial region ranging from facilitate diagnosis to image guidance of operative and surgical procedures. Moreover, this technique is able to produce images in axial, sagittal, frontal planes.

The potential scope of clinical applications for cone-beam imaging is vast and currently has been shown to be particularly useful in the following dental and maxillofacial areas: ${ }^{5}$ i) Investigation of jaw pathology including cysts, tumours and fibro-osseous lesions; ii) Investigation of the paranasal sinuses; iii) Investigation of the bony components of the TMJ; iv) Pre- and post-implant assessment; v) Orthodontic assessment, both dental development and skeletal base relationship; vi) Assessment of wisdom teeth, in particular their relationship to the inferior dental canal; vii) Evaluation of facial trauma; viii) Periodontal assessment.

The dental CBCT is recommended for: assessment of bone support for the application of dental implants; TMJ's analysis to diagnose degenerative bone changes; examination of teeth and facial structures to start orthodontic treatment; proximity viewing of wisdom molars to lower mandibular canal, prior extractions; diagnosis of cysts, tumors or infections of the teeth and jaw bones.

There are four components to CBCT image acquisition: ${ }^{6}$ i) $X$-ray generation: continuous or pulsed $\mathrm{x}$-ray beam and charged couple device detectors moving synchronously around the fixed fulcrum within the patient's head; ii) Image detection: it is determined by individual volume elements or voxels produced from the volumetric data set. CBCT units provide voxel resolutions that are isotropic (equal in all 3 dimensions); iii) Image reconstruction: the processing of acquired projection frames to the
Correspondence: Anuroopa Pudukulangara Nair, Department of Periodontics,

Rajarajeshwari dental college \& hospital, Bangalore - 560060, India.

Tel. +91.998.671.9039.

E-mail: anuganga04@gmail.com

Key words: CBCT, anatomical structure, diagnosis, low dose, tomography, quantitative analyses, bone density.

Received for publication: 6 September 2011

Revision received: 20 0ctober 2011

Accepted for publication: 21 October 2011.

This work is licensed under a Creative Commons Attribution NonCommercial 3.0 License (CC BYNC 3.0).

(C) Copyright K.G. Panicker et al., 2011

Licensee PAGEPress, Italy

Surgical Techniques Development 2011; 1:e31

doi:10.4081/std.2011.e31

volumetric dataset is done on the personal computer which is called as reconstruction; iv) Image display: the compilation of all available voxels is presented to the clinician on the computer screen as secondary reconstructed images in three orthogonal planes.

It is beyond the scope of this article to detail these elements; however, it is important to recognize that the specifications of currently available systems reflect proprietary variations in these parameters.

\section{Types of computed \\ tomography scanners}

Computed tomography can be divided into 2 categories based on acquisition x-ray beam geometry; namely: fan beam and cone beam.

In fan-beam scanners, an x-ray source and solid-state detector are mounted on a rotating gantry. Data are acquired using a narrow fanshaped $\mathrm{x}$-ray beam transmitted through the patient. The patient is imaged slice-by-slice, usually in the axial plane, and interpretation of the images is achieved by stacking the slices to obtain multiple 2D representations. The linear array of detector elements used in conventional helical fan-beam CT scanners is actually a multi-detector array. This configuration allows multi-detector CT (MDCT) scanners to acquire up to 64 slices simultaneously, considerably reducing the scanning time compared with single-slice systems and allowing generation of 3D images at substantially lower doses of radiation than single detector fan-beam CT array.

\section{Cone-Beam computed}

\section{tomography technology}

CBCT scanners are based on volumetric tomography, using a 2D extended digital array providing an area detector. This is combined 
with a 3D x-ray beam. The cone-beam technique involves a single $360^{\circ}$ scan in which the $\mathrm{x}$-ray source and a reciprocating area detector synchronously move around the patient's head, which is stabilized with a head holder. At certain degree intervals, single projection images, known as basis images, are acquired. These are similar to lateral cephalometric radiographic images, each slightly offset from one another. This series of basis projection images is referred to as the projection data. Software programs incorporating sophisticated algorithms including back-filtered projection are applied to these image data to generate a 3D volumetric data set, which can be used to provide primary reconstruction images in 3 orthogonal planes (axial, sagittal and coronal). ${ }^{6}$

\section{Advantages of Cone-Beam com- puted tomography}

The use of CBCT technology in clinical practice provides a number of potential advantages for maxillofacial imaging compared with conventional $\mathrm{CT}{ }^{6}$

\section{X-ray beam limitation}

Reducing the size of the irradiated area by collimation of the primary $x$-ray beam to the area of interest minimizes the radiation dose. Most CBCT units can be adjusted to scan small regions for specific diagnostic tasks. Others are capable of scanning the entire craniofacial complex when necessary.

\section{Image accuracy}

The volumetric data set comprises a 3D block of smaller cuboid structures, known as voxels, each representing a specific degree of $x$-ray absorption. The size of these voxels determines the resolution of the image. In conventional $\mathrm{CT}$, the voxels are anisotropic - rectangular cubes where the longest dimension of the voxel is the axial slice thickness and is determined by slice pitch, a function of gantry motion. Although CT voxel surfaces can be as small as $0.625 \mathrm{~mm}$ square, their depth is usually in the order of 1-2 mm. All CBCT units provide voxel resolutions that are isotropic - equal in all 3 dimensions. This produces submillimetre resolution (often exceeding the highest grade multi-slice $\mathrm{CT}$ ) ranging from $0.4 \mathrm{~mm}$ to as low as $0.125 \mathrm{~mm}$ (Accuitomo).

\section{Rapid scan time}

Because CBCT acquires all basis images in a single rotation, scan time is rapid (10-70 seconds) and comparable with that of medical spiral MDCT systems. Although faster scanning time usually means fewer basis images from which to reconstruct the volumetric data set, motion artifacts due to subject movement are reduced.

\section{Dose reduction}

Published reports indicate that the effective dose of radiation (average range 36.9-50.3 microsievert $[\mu \mathrm{Sv}])^{7}$ is significantly reduced by up to $98 \%$ compared with conventional fanbeam CT systems (average range for mandible 1.320-3.324 $\mu \mathrm{Sv}$; average range for maxilla

1.031-1.420 $\mu \mathrm{Sv}){ }^{8}$ This reduces the effective patient dose to approximately that of a film-based periapical survey of the dentition (13-100 $\mu \mathrm{Sv})$ or 4-15 times that of a single panoramic radiograph $(2.9-11 \mu \mathrm{Sv}){ }^{9}$

\section{Display modes unique to maxillofacial imaging}

Reconstruction of CBCT data is performed natively by a personal computer. In addition, software can be made available to the user, not just the radiologist, either via direct purchase or innovative per use licence from various vendors (e.g., Imaging Sciences International). This provides the clinician with the opportunity to use chair-side image display, real-time analysis and MPR modes that are task specific. Because the CBCT volumetric data set is isotropic, the entire volume can be reoriented so that the patient's anatomic features are realigned. In addition, cursor-driven measurement algorithms allow the clinician to do realtime dimensional assessment.

\section{Reduced image artifact}

With manufacturer's artifact suppression algorithms and increasing number of projections, CBCT images can result in a low level of metal artifact, particularly in secondary reconstructions designed for viewing the teeth and jaws. ${ }^{7}$

\section{Limitations}

i) Research into cone-beam imaging has to meet the challenge of rapid changes in both hard- and soft-ware technology; ii) The equipment itself is changing in order to meet the clinical requirements reported to manufacturers, who in turn have markets to consider. However, there are intrinsic limitations in the technique which mean, in some circumstances, other forms of dental imaging would be more appropriate. Caries and teeth adjacent to amalgam and other dense prosthetic restorations are not well imaged by cone-beam technology owing to beam hardening and streak artefact. Even gutta percha may give rise to streak artefact and appear as dense as amalgam might on conventional CT. This should be borne in mind when assessing a potential site for implants adjacent to rootfilled teeth; iii) Both lamina dura and bony detail can be better assessed on periapical radiographs compared to cone-beam; ${ }^{10}$ iv) In order to acquire an undistorted image with conebeam imaging, it is essential that the patient's head is kept still during the gantry rotation. As with dental panoramic tomography, patient movement can limit the technique for very young children, those unable to stay still or with movement disorders; v) Interestingly, to those not used to working with 3-D volumes, radiological interpretation can be difficult when using a smaller field of view, as it is easy to become disoriented when scrolling through the images, as points of reference such as normal dental landmarks, or anomalous anatomy can make orientation difficult; vi) CBCT based on an image intensifier may allow the periphery of the image to be distorted; vii) CBCT gives little in the way of soft tissue detail and, and in no way compares to those capable of conventional CT. This, precludes the technique in the assessment of head and neck malignancy where evaluating the soft tissue extent of the lesion is crucial.

\section{Clinical applications of Cone-Beam computed tomography in dentistry}

With CBCT technology, all radiographic images can be taken in less than a minute. Dental clinicians can have the diagnostic quality of periapical radiographs, panoramic radiographs, cephalograms, occlusal radiographs, and TMJ images at their disposal, along with views that cannot be produced with regular radiographic machines such as axial and cross-sectional views. A number of clinical applications have already been reported in the literature. ${ }^{11}$

\section{Impacted teeth}

Impacted maxillary cuspids have been reported to be distributed as $85 \%$ palatal and $15 \%$ buccal. The CBCT allows for a more precise analysis of the extent of the pathology related to the ectopic tooth. Clinical reports using 3-dimensional imaging have shown that the incidence of root resorption of teeth adjacent to impacted teeth is greater than previously thought. CBCT images can be used to locate the precise position of ectopic cuspids and to design treatment strategies that would result in less invasive surgical intervention. Computer- and image-guided surgical exposure allows for less invasive surgery, smaller incisions, more conservative flap design, and overall reduced morbidity associated with the surgery. ${ }^{12,13}$ (Figures 1- 3).

\section{Pathology}

Another use of CBCT is the location of (oral) pathologic lesions such as periapical cysts. CBCT has been evaluated for the detection of carious lesions and has shown better results than F-speed film in assessing the depth of proximal lesions. ${ }^{14}$

\section{Airway analysis}

CBCT technology provides a major improvement for evaluation of the airway, allowing for 


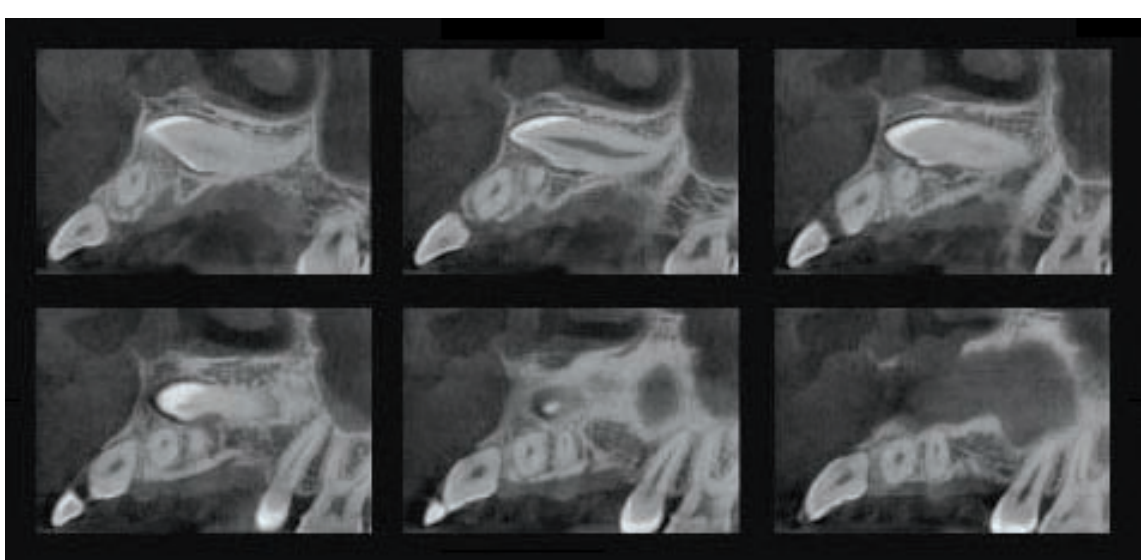

Figure 1. Impacted bilateral Maxillary canines.

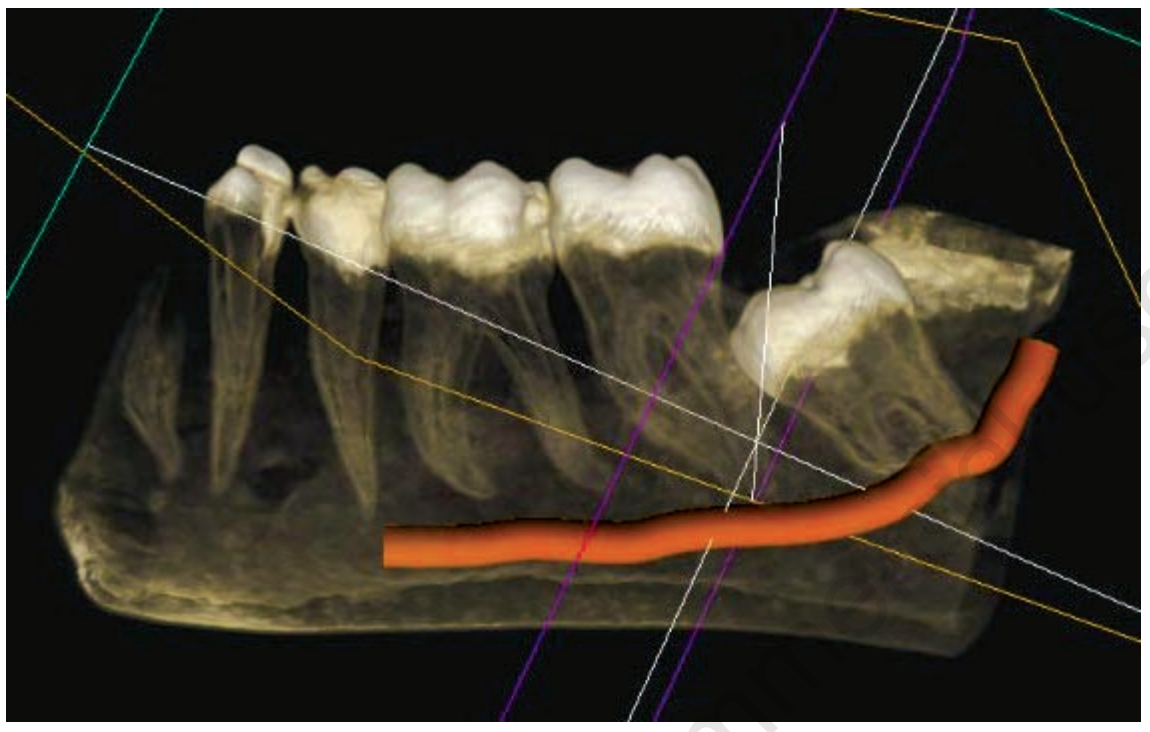

Figure 2. Close proximity of the impacted lower third molar to the inferior alveolar canal.

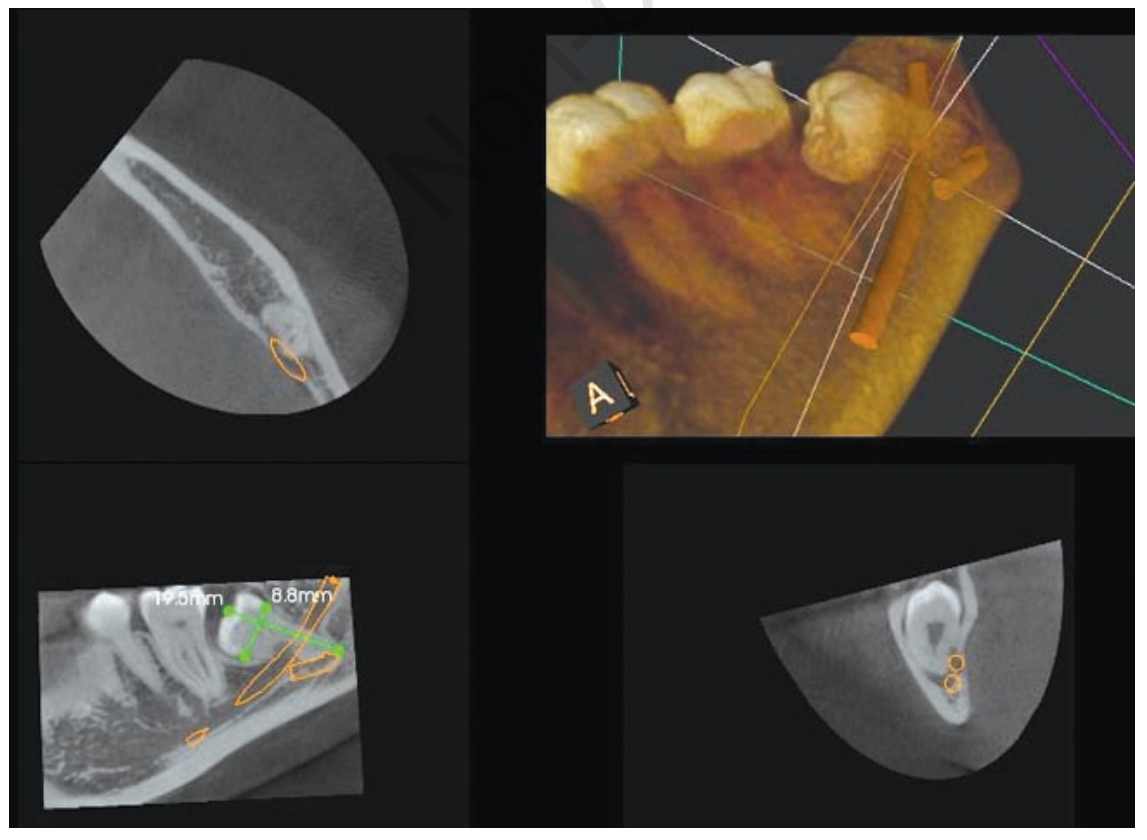

Figure 3. Split or twinned inferior alveolar canal with impacted lower third molar. 3-dimensional and volumetric determinations. Airway analysis conventionally has been carried out by using lateral cephalograms. Threedimensional airway analysis will be useful for the understanding of more complex conditions such as obstructive sleep apnea (OSA) and enlarged adenoids. ${ }^{15}$

\section{Implant planning and bone quality assessment}

Implantologists have long appreciated the value of 3- dimensional imaging. Conventional CT scans are used to assess the osseous dimensions, bone density, and alveolar height, especially when multiple implants are planned. Locating landmarks and anatomy such as the inferior alveolar canal, maxillary sinus, and mental foramen occurs more accurately with a CT scan. The use of the third dimension has improved the clinical success of implants and their associated prostheses, and led to more accurate and aesthetic outcomes. ${ }^{16}$ (Figure 4-7).

CBCT has been in use in implant therapy and may be employed in orthodontics for the clinical assessment of bone graft quality following alveolar surgery in patients with cleft lip and palate. The images produced provide more precise evaluation of the alveolus. This technology can help the clinician determine if the patient should be restored or if teeth should be moved orthodontically into the repaired alveolus. ${ }^{17}$

\section{Location of anatomic structures}

Anatomic structures such as the inferior alveolar nerve, maxillary sinus, mental foramen, and adjacent roots are easily visible using CBCT. The CBCT image also allows for precise measurement of distance, area, and volume. Using these features, clinicians can feel confident in the treatment planning for sinus lifts, ridge augmentations, extractions, and implant placements.

\section{Temporomandibular joint morphology}

CBCT imaging of the temporomandibular joint has been evaluated and compared to other methods. The CBCT showed greater sensitivity and accuracy than the helical CT in the identification of mandibular condyle abnormalities. Recent 3-dimensional studies have attempted to understand how the condyle remodels, and preliminary data suggest that much of the condylar remodeling is a direct result of the surgical procedure. ${ }^{18}$

\section{Periodontal applications}

Usefulness of CBCT for periodontal applications is still in progress. Field of interest for the use in periodontology would be the diag- 
nostic and quantitative measurements of soft tissue and alveolar bone levels in three dimensions, imaging of periodontal intrabony defects, dehiscence and fenestration defects, diagnosis of furcation-involved molars, and implant site imaging. CBCT is a superior technique when buccal and lingual defects cannot be diagnosed with radiography. Soft tissue CBCT (ST-CBCT), ${ }^{19}$ is used to visualize and

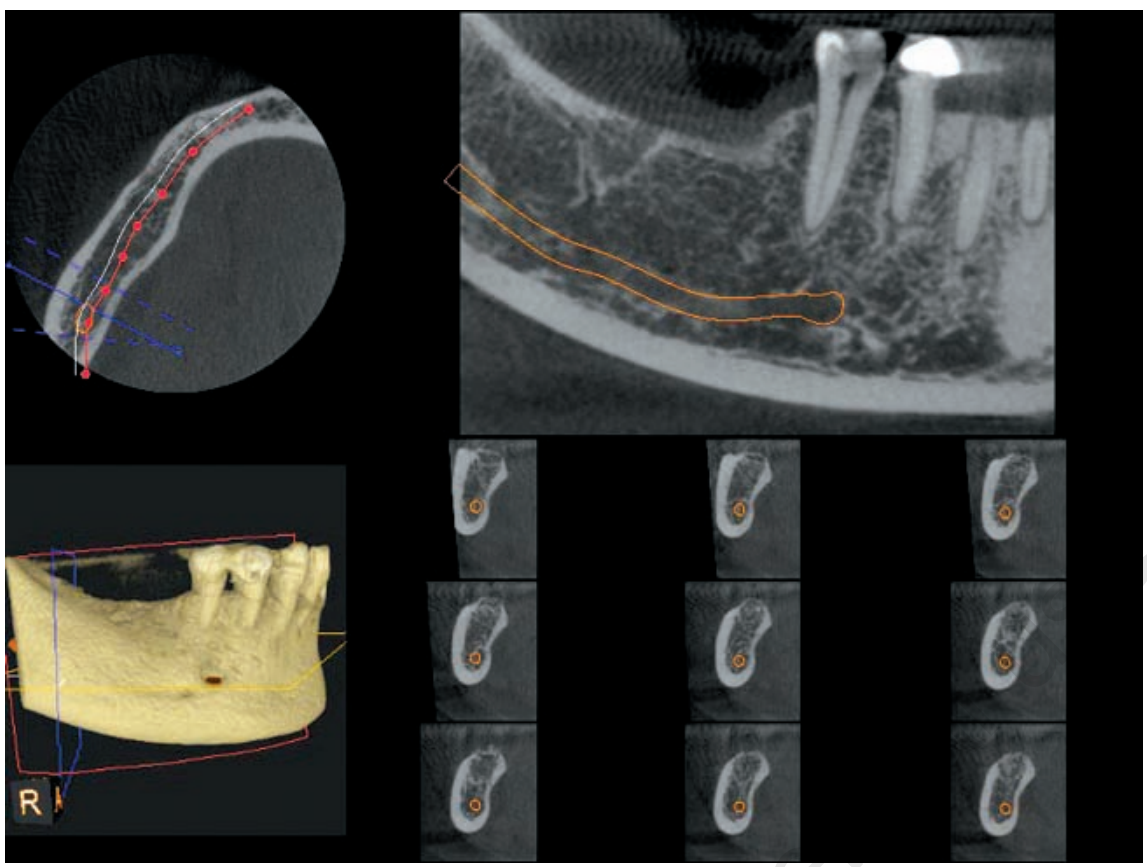

Figure 4. Tracing the course of the inferior alveolar canal.

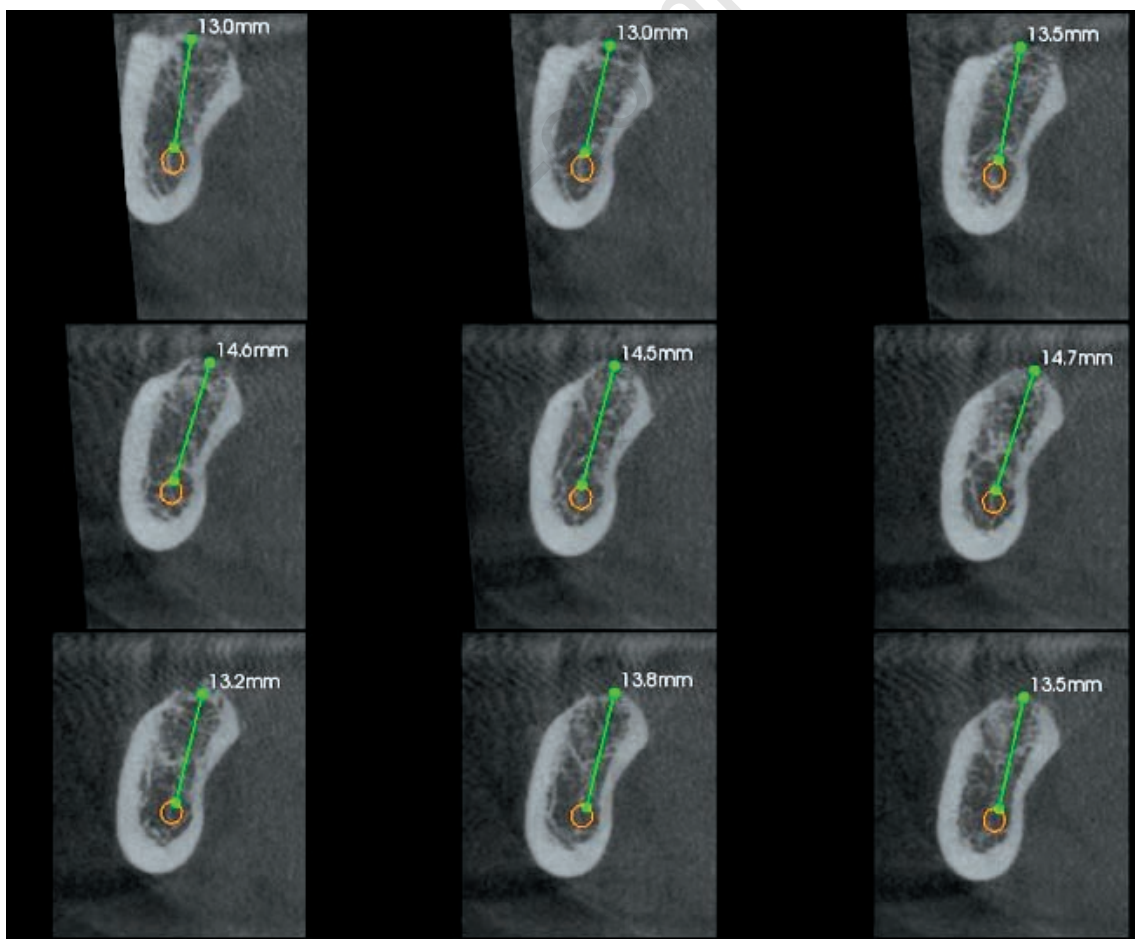

Figure 5. Bone Height measurements on the mandible for implant planning. precisely measure distances corresponding to the hard and soft tissues of the periodontium and dentogingival attachment apparatus. With this simple and noninvasive technique, clinicians are able to determine the relationships bone crest; ii) gingival margin and the cemento-enamel junction (CEJ), and iii) CEJ and facial bone crest. between: i) gingival margin and the facial
The width of the facial and palata//ingual alveolar bone and the width of the facial and palata//lingual gingival also can be measured.

\section{Discussion}

Cone-beam imaging, sometimes referred to as digital volume tomography, is one of the most exciting developments in dental and maxillofacial radiology and, owing to its versatility, will almost certainly become an increasingly popular form of imaging available in dental practice. ${ }^{5}$

Interpretation demands an understanding of the spatial relations of bony anatomical elements and a comprehensive pathological knowledge of the various maxillofacial structures involved. Obviously, this information can extend beyond purely the dento-alveolar complex. The obvious potential for missed occult pathology with these units does, if nothing else increases the risk of litigation. A recent study using CBCT showed $24.6 \%$ had incidental findings. It would be in the patient's best interest that an imaging specialist with optimal knowledge of this area view the total volume obtained during image acquisition. ${ }^{5}$

Kobayashi et al. ${ }^{20}$ confirmed the superiority of PSR 9000 cone beam CT to spiral CT in terms of spatial resolution on cross-sectional images. Similar findings were reported when comparing images from an anthropomorphic phantom taken by both the 3DX Multi Image Micro CT (J. Morita) and the multidetector Aquilion Multi-Slice CT (Toshiba Medical Co Ltd, Tokyo, Japan). The superiority of the 3DX cone beam device in the images' resolution was demonstrated by means of a high-resolution score of the periodontal ligament space and the lamina dura.

Honda et al. ${ }^{21}$ compared helical CT with the Ortho- CT and reported that the image quality obtained with the Ortho-CT far surpassed that of the helical CT. To achieve accurate information and sufficient detail for preoperative planning of implant surgery, image quality of the different devices should be analyzed.

The problem is exacerbated by the fact that cone-beam imaging uses ionizing radiation doses exceeding any other existing form of dental imaging. The basic tenets of ALARA and maximizing the benefit/risk ratio to the patient still apply when selecting cases for imaging. With increasing potential use of cone-beam imaging for a variety of clinical situations, guidelines need to be developed indicating best practice. Dental undergraduates will require training in the interpretation and limitations of cone-beam CT. The ability to export data into software packages, such as Simplant/Materialise and Nobel Biocare and their manipulation should also form part of 
postgraduate teaching curricula, building on an undergraduate exposure to this modality.

\section{Conclusions}

Cone Beam Computed Tomography (CBCT) scans have been well established as a valuable tool in the orthodontist and surgeon's 3D toolkit. A single scan not only provides an overlap-

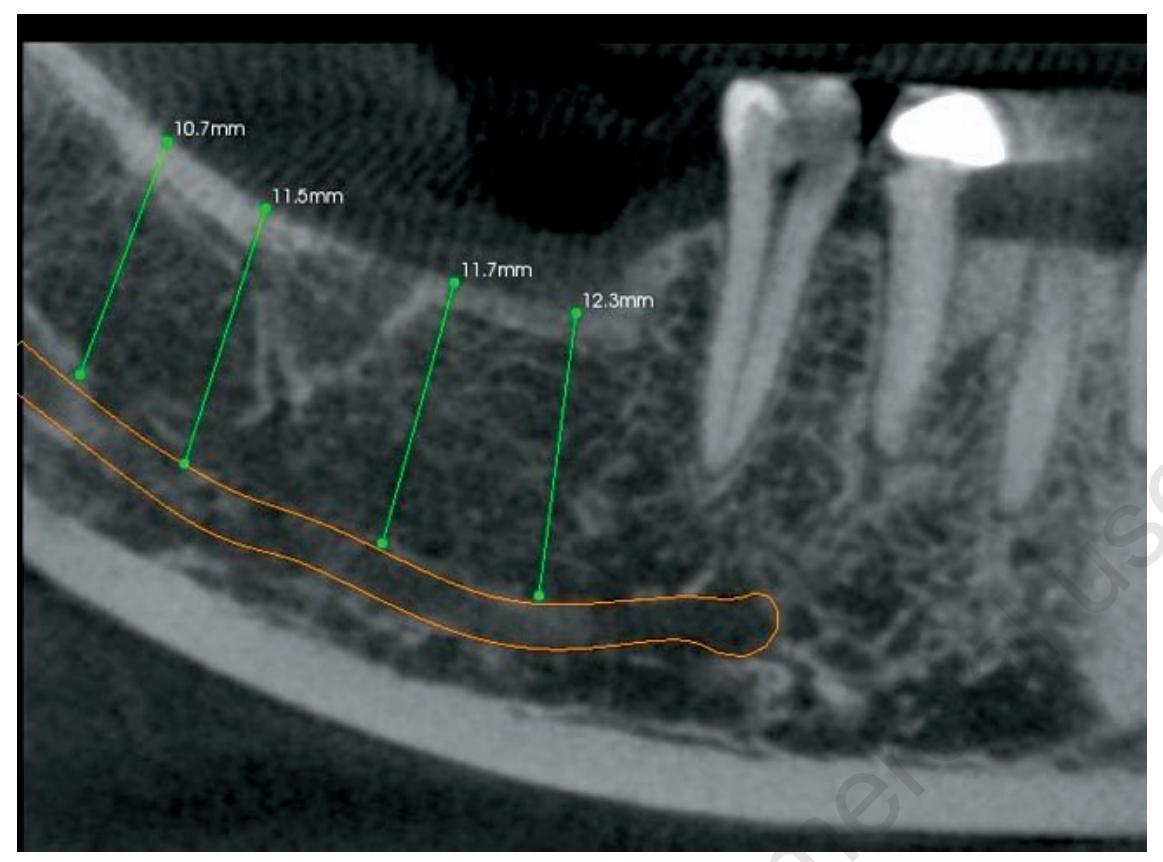

Figure 6. Bone Height measurements on the mandible for implant planning.

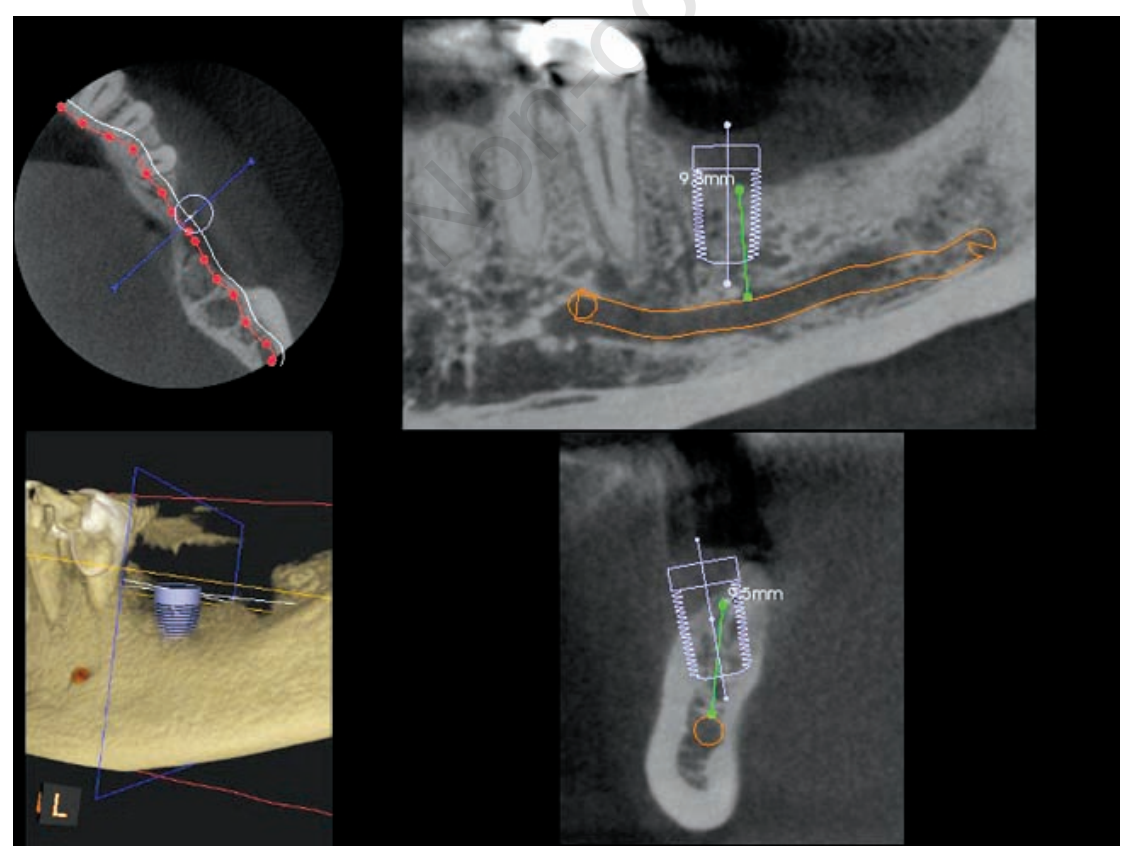

Figure 7. Implant simulation. allows detailed evaluation of the maxillofacial structures in thin axial, coronal and sagittal slices. It provides clear images of highly contrasted structures and is extremely useful for evaluating bone. Although limitations currently exist in the use of this technology for softtissue imaging, efforts are being directed toward the development of techniques and software algorithms to improve signal-to-noise ratio and increase contrast. Increasing avail- free 3D visualization of the skull but also ability of this technology provides the practitioner with a modality that is extending maxillofacial imaging from diagnosis to image guidance of operative and surgical procedures.

\section{References}

1. Sara Samur. Cone Beam Computed Tomography In Dentistry. AD0 J Clin Sci 2009;3;346-51.

2. Mozzo P, Procacci C, Tacconi A, et al. A new volumetric CT machine for dental imaging based on the cone-beam technique: Preliminary results. Eur Radiol 1998;8: 1558.

3. Arai Y, Tammisalo E, Iwai K. Development of a compact computed tomographic apparatus for dental use. Dentomaxillofac Radiol 1999;28:245.

4. Hashimoto K, Yoshinori A, Kazui I. A comparison of a new, limited cone beam computed tomography machine for dental use with a multi- detector row helical CT machine. Oral Surg Oral Med Oral Pathol Oral Radiol Endod 2003;95:371-5.

5. Mcleod I, Heath N. Cone-Beam Computed Tomography (CBCT) in Dental Practice. Dent Update 2008;35:590-8

6. Scarfe WC, Farman AG, Sukovic P. Clinical Applications of Cone-Beam Computed Tomography in Dental Practice. J Can Dent Assoc 2006;72:75-80

7. Hu H, He HD, Foley WD, Fox SH. Four multidetector-row helical CT: image quality and volume coverage speed. Radiology 2000;215:55-62.

8. Cohnen M, Kemper J, Mobes 0, et al. Radiation dose in dental radiology. Eur Radiol 2002;12:634-7.

9. Ludlow JB, Davies-Ludlow LE, Brooks SL. Dosimetry of two extraoral direct digital imaging devices: NewTom cone beam CT and Orthophos Plus DS panoramic unit. Dentomaxillofac Radiol 2003;32:229-34.

10. Patel S, Dawood A, Ford TP, Whaites E. The potential applications of cone beam tomography in the management of endodontics problems. Int Endo J 2007;40: 818-30.

11. Palomo JM, Kau CH, Bahl L, Hans MG. Three-dimensional cone beam Computerized tomography in dentistry. Int Dent SA 2008;9:40-9.

12. Walker L, Enciso R, Mah J. Three dimensional localization of maxillary canines with cone-beam computed tomography. Am J Orthod Dentofacial Orthop 2005;128: 418-23.

13. Mah J, Enciso R, Jorgensen M. Management of impacted cuspids using 3-D volumetric imaging. J Calif Dent Assoc 2003; 31:835-41. 
14. Akdeniz BG, Grondahl HG, Magnusson B. Accuracy of proximal caries depth measurements: comparison between limited cone beam computed tomography, storage phosphor and film radiography. Caries Res 2006;40:202-7.

15. Aboudara CA, Hatcher D, Nielsen IL, et al. A three-dimensional evaluation of the upper airway in adolescents. Orthod Craniofac Res 2003;6 Suppl 1:173-5.

16. Hatcher DC, Dial C, Mayorga C. Cone beam CT for pre-surgical assessment of implant sites. J Calif Dent Assoc 2003;31:
825-33.

17. Hamada Y, Kondoh T, Noguchi K, et al. Application of limited cone beam computed tomography to clinical assessment of alveolar bone grafting: a preliminary report. Cleft Palate Craniofac J 2005;42: 128-137.

18. Bailey LJ, Cevidanes LH, Proffit WR. Stability and predictability of orthognathic surgery. Am J Orthod Dentofacial Orthop 2004;126:273-7.

19. Mohan R, Singh A, Gundappa M. Threedimensional imaging in periodontal diag- nosis - Utilization of cone beam computed tomography. Indian Soc Periodontol 2011;15:11-17.

20. Kobayashi K, Shimoda S, Nakagawa Y, Yamamoto A. Accuracy in measurement of distance using limited cone-beam computerized tomography. Int J Oral Maxillofac Implants 2004;19:228-31.

21. Honda K, Arai Y, Iwai K, et al. Fundamental efficiency of new-style limited cone-beam CT (3DX). Comparison with Helical CT. Jpn J Tomogr 2000;27:17-22. 